\title{
La evidencia en tiempos de coronavirus (COVID-19)
}

\author{
Evidence in times of coronavirus (COVID-19)
}

Martin Cañás ${ }^{a, b}$, Martin A Urtasun ${ }^{a, b}$

\begin{abstract}
Resumen
La pandemia de COVID-19 está generando información epidemiológica y clínica en una escala sin precedentes para una enfermedad de reciente aparición. Aunque ya se han iniciado numerosos ensayos clínicos de fármacos antiguos y nuevos como potenciales antivirales específicos, la mayor parte de la información publicada hasta ahora carece de los controles básicos para la evaluación de la eficacia de un medicamento. Los medios de comunicación amplifican estos resultados preliminares y suman presión a los médicos asistenciales y a los decisores de políticas públicas. Este artículo revisa las pruebas disponibles sobre los cuatro tratamientos antivirales específicos más prometedores: hidroxicloroquina, lopinavir/ritonavir, remdesvir e interferones alfa y beta. Se comprueba en todos ellos que no hay demostración suficiente de eficacia como para recomendar su uso fuera de una investigación experimental adecuadamente controlada. En el uso individual de un medicamento no hay forma de saber si está beneficiando o perjudicando al paciente. Es erróneo asumir que la eventual curación se debe al fármaco y un mal desenlace debe atribuirse a la enfermedad. Sólo la comparación entre grupos de pacientes asignados al tratamiento experimental o a un control adecuado permite conocer la eficacia y seguridad de las intervenciones. El desafío es conciliar la urgencia de actuar con la generación de nuevos conocimientos. Aunque no resulta sencillo organizar ensayos clínicos en este contexto, las instituciones pueden sumarse a los proyectos en marcha a nivel nacional e internacional. El uso de estos fármacos debe considerarse experimental, por lo que es necesario obtener el consentimiento informado del paciente.
\end{abstract}

\section{Abstract}

The COVID-19 pandemic is generating epidemiological and clinical information on an unprecedented scale for a newly emerging disease. Although numerous clinical trials of old and new drugs as potential specific antivirals have already been started, most of the information published so far lacks basic controls for evaluating drug efficacy. The media amplify these preliminary results and add pressure to doctors and policymakers. This article reviews the available evidence for the four most promising specific antiviral treatments: hydroxychloroquine, lopinavir / ritonavir, remdesvir, and alpha and beta interferons. The findings show that none of them has sufficient demonstration of efficacy to recommend its use outside of the adequately controlled experimental study. In the individual use of a drug there is no way of knowing if it is benefiting or harming the patient. It is wrong to assume that the eventual cure is due to the drug and a poor outcome must be attributed to the disease. Only the comparison between groups of patients assigned to the experimental treatment or to an adequate control can establish the efficacy and safety of the interventions. The challenge is to reconcile the urgency to act with the generation of new knowledge. Although it is not easy to organize clinical trials in this context, the institutions can join the ongoing projects at the national and international levels. The use of these drugs should be considered experimental, so it is necessary to obtain the informed consent of the patient.

Palabras clave: Infecciones por Coronavirus, Medicina Basada en la Evidencia. Keywords: Coronavirus Infections, Evidence-Based Medicine.

Cañás M, Urtasun MA. La evidencia en tiempos de coronavirus (COVID-19). Evid Actual Pract Ambul. 2020;23(2):e002057

\section{La evidencia en tiempos de coronavirus}

La pandemia de COVID-19 se acompaña de un flujo sin precedentes de información científica. La publicación en línea de investigaciones sobre el coronavirus, expeditiva y de acceso gratuito, inunda la portada de las principales revistas médicas.

A partir de las experiencias de los primeros países afectados se definieron muchos aspectos epidemiológicos y clínicos de la enfermedad. En la búsqueda de tratamiento específico para el Sars-CoV2 se recurrió a los datos de investigación básica y a los escasos precedentes de estudios clínicos con otros coronavirus. Así se han propuesto más de diez potenciales tratamientos y se han iniciado ensayos clínicos para evaluarlos. También se publican experiencias terapéuticas de series de casos sin grupo control, con todas las limitaciones conocidas de este tipo de estu$\operatorname{dios}^{1,2}$

Mientras aguardamos los resultados de los ensayos clínicos, los pacientes se internan con cuadros pulmonares severos, que en la tercera o cuarta parte de los casos requieren cuidados intensivos, a menudo con asistencia respiratoria mecánica. Frente a este grave panorama ¿debemos ofrecer tratamientos sin pruebas suficientes de su relación beneficio-riesgo, en base a datos preliminares? ¿O esperar a la publicación de las pruebas? ¿O administrarlos sólo en el contexto de una investigación apropiadamente diseñada? Los profesionales asistenciales y las autoridades de salud pública están demandando al mundo académico respuestas urgentes a estas preguntas.

\section{Tratamiento antiviral específico para SARS-CoV-2}

Desde el inicio de la pandemia, un gran número de pacientes han recibido terapias de uso compasivo y fuera de etiqueta, incluyendo la cloroquina, la hidroxicloroquina (HCQ), la azitromicina, la combinacion lopinavir-ritonavir (LPV/r), el favipiravir, el remdesivir, la ribavirina, el interferón, el plasma convaleciente, los corticoides sistémicos y los inhibidores anti-IL-6 (tocilizumab y otros). Sin embargo, a la fecha no hay resultados concluyentes que demuestren la eficacia de alguno de estos tratamientos y su relación beneficio-riesgo ${ }^{1,2}$.

\footnotetext{
a Área Farmacología, Fundación FEMEBA, Argentina..

b Instituto de Ciencias de la Salud, Universidad Nacional Arturo Jauretche, Argentina. farmacol@femeba.org.ar, martinurtasun@yahoo.com.ar
} 
Entre los más mencionados en los medios de comunicación, y ya incluidos en protocolos de rescate de varios países, se encuentran HCQ (sola o asociada con azitromicina), LPV/r, remdesivir e interferón beta. Los dos primeros aparecen en las recomendaciones del Ministerio de Salud de la Nación de Argentina $(\mathrm{MSN})^{3}$ y los cuatro fármacos (solos o asociados) se estudian en el megaensayo Solidarity ${ }^{4}$, organizado por la Organización Mundial de la Salud (OMS). Revisaremos brevemente las pruebas disponibles a la fecha, conscientes de que este resumen puede quedar desactualizado en pocas semanas.

\section{Hidroxicloroquina}

Además de su eficacia para la malaria, la amebiasis y afecciones inflamatorias crónicas, como la artritis reumatoide o el lupus eritematoso, la HCQ tiene actividad antiviral in vitro. Sin embargo, la eficacia clínica en enfermedades virales no está establecida ${ }^{2}$. Los mecanismos de acción incluyen la glucosilación de la enzima convertidora de angiotensina-2, que disminuye el ingreso del SARS-CoV-2 a las células; el deterioro de la acidificación de los endosomas, que interfiere el tráfico viral en las células; y los efectos inmunomoduladores, que pueden atenuar la tormenta de citoquinas en la enfermedad severa. Sin embargo, debe recordarse que las acciones inmunosupresoras pueden ser perjudiciales en la enfermedad viral ${ }^{2,4}$.

Un informe rápido de la Red Argentina de Evaluación de Tecnología Sanitaria (RedARETS) concluye que no hay certezas de la eficacia de HCQ sobre desenlaces clínicamente relevantes en pacientes con infección por SARS-CoV-2. La escasa evidencia clínica existente, calificada como de muy baja confianza, muestra que la $\mathrm{HCQ}$ podría ser más eficaz que el tratamiento habitual para negativizar la eliminación viral a los 6 días, y la combinación de HCQ con azitromicina podría ser más eficaz que HCQ sola. En cuanto a su seguridad, no se halló evidencia de efectos adversos severos por el uso a corto plazo de HCQ; mientras que, en tratamientos prolongados, se destaca especialmente la toxicidad cardíaca, a menudo irreversible, y que puede generar arritmias letales e insuficiencia cardíaca, entre otras complicaciones ${ }^{5}$. Otra revisión sistemática concluye que está justificada la realización de ensayos clínicos en este contexto, pero que el tratamiento debe considerarse experimental y no recomienda el uso clínico generalizado ${ }^{6}$.

Las guías actuales del MSN reconocen el carácter condicional del uso de HCQ, por la debilidad de las pruebas existentes. Por tratarse de un uso fuera de prospecto, se debe obtener el consentimiento informado del paciente o su representante ${ }^{3}$.

Revisemos brevemente los estudios disponibles. En un pequeño ensayo clínico aleatorizado, realizado en Shangai, el aclaramiento viral nasofaríngeo en el día 7 no fue diferente con $\mathrm{HCQ}$ frente al tratamiento estándar ${ }^{7}$. Un estudio francés, que atrajo mucha atención académica y mediática, evaluó la presencia del virus en la vía aérea en una comparación no aleatorizada entre 26 pacientes que recibieron $\mathrm{HCQ}$ y un grupo control, reclutado de manera diferente ${ }^{8}$. Se observó la negativización virológica al $6^{\circ}$ día en $70 \%$ de los tratados con HCQ y en $100 \%$ de 6 pacientes que recibieron además azitromicina. Debido a la ausencia de controles comparables, este resultado no es concluyente ${ }^{9}$. En un giro inesperado, la International Society of Antimicrobial Chemotherapy, editora de la revista donde se publicó el estudio francés, publicó una declaración planteando que "...el artículo no cumple con el estándar esperado de la Sociedad, especialmente en relación con la falta de mejores explicaciones de los criterios de inclusión y del triage de pacientes para garantizar la seguridad del paciente" (traducción de los autores) ${ }^{10}$. El mismo grupo francés informó que $93 \%$ de una serie de 80 pacientes internados tratados con HCQ más azitromicina negativizó la PCR en el $8^{\circ}$ día de tratamiento. La falta de un grupo control y de resultados clínicos limita la posibilidad de establecer conclusiones en cuanto a su eficacia ${ }^{11}$. Por otro lado, un ensayo clínico aleatorizado de 62 pacientes internados que no estaban en estado crítico sugiere que $400 \mathrm{mg}$ diarios de HCQ durante 5 días aceleran modestamente la desaparición de los síntomas y la mejoría radiológica ${ }^{12}$. Adicionalmente, a la fecha de edición final de este artículo se han dado a conocer otros tres estudios de HCQ aun no aceptados para su publicación, sin resultados concluyentes ${ }^{13-15}$

No hay todavía estudios sobre la eficacia y la seguridad de HCQ en profilaxis post-exposición ni en la prevención del contagio en personal de salud ${ }^{16}$. Dentro de los riesgos conocidos, las interacciones con fármacos que prolonguen el intervalo QT son especialmente preocupantes. La Sociedad Argentina de Terapia Intensiva ha detallado las contraindicaciones y precauciones necesarias para su uso en este contexto ${ }^{17}$, que también se reflejan en las guías del MSN ${ }^{3}$

\section{Lopinavir / ritonavir}

La combinación de los agentes antivirales LPV/r se utiliza en el tratamiento del VIH: el lopinavir es un inhibidor eficaz de la proteasa viral y el ritonavir aumenta su eficacia al prolongar la vida media.

El informe rápido de la RedARETS sobre la eficacia de LPV/r en COVID-19 encuentra evidencia de baja certeza de que, en pacientes con diagnóstico reciente, podría lograrse una reducción modesta de la mortalidad en infecciones leves y una reducción moderada de la mortalidad en infecciones graves. En ambos casos, el intervalo de confianza del efecto anticipado incluye la ausencia de eficacia. Por otra parte, no se observó un aumento de efectos adversos severos en esta combinación ${ }^{18}$. El informe señala además la preocupación por el costo elevado del tratamiento y su posible efecto negativo sobre la equidad entre diferentes ámbitos de atención de la salud ${ }^{18}$.

Las recomendaciones del MSN, reconociendo la baja calidad de la evidencia, proponen utilizarlo en casos de neumonía grave, con o sin HCQ asociada, y como una opción a la HCQ en casos de neumonía leve ${ }^{3}$. Tal como se mencionó para la HCQ, este uso se consideran fuera de prospecto y requiere el consentimiento informado del paciente o su representante. El estudio más importante de LPV/r es un ensayo clínico con 199 pacientes internados con cuadros graves de COVID-19, asignados al azar a recibir la combinación durante 14 días o la atención estándar. La gravedad de los pacientes se refleja en una mortalidad cercana al $25 \%$. No hubo diferencia significativa en el desenlace clínico primario ni en la eliminación viral. Hubo una reducción de la mortalidad que no alcanzó la significación estadística. Aunque los efectos adversos fueron frecuentes en el grupo de tratamiento, los eventos graves fueron más comunes en el grupo control ${ }^{19}$. Queda por establecer el efecto de la combinación en casos de enfermedad menos severa $^{20}$

EI LPV/r causa efectos adversos gastrointestinales, infección respiratoria alta, dislipidemia, disglucemia, bloqueo aurículoventricular y prolongación del intervalo QT. Ademas, tiene numerosas interacciones farmacológicas ${ }^{21}$.

\section{Remdesivir}

Este análogo nucleotídico fue desarrollado para el tratamiento de la infeccion por el virus del Ébola. Tiene efecto antiviral de amplio espectro por inhibir la ARN polimerasa dependiente de ARN. Ha demostrado actividad in vitro e in vivo en modelos animales de MERS y SARS; frente al coronavirus causante del MERS ha mostrado mejores resultados que el tratamiento con LPV/r más interferón $\beta 1 b^{22}$.

Están en marcha dos ensayos clínicos aleatorizados y controlados con tratamiento estándar, uno en pacientes con enferme- 
dad moderada y otro con enfermedad grave, cuyos resultados se esperan en abril.

Por tratarse de un medicamento experimental, su perfil de efectos adversos no es bien conocido; los principales son la hipotensión infusional y las molestias gastrointestinales. Se deben tener en cuenta los criterios de exclusión de los ensayos en curso: falla multiorgánica, ventilación mecánica, ALT o AST mayor a 5 veces el límite superior normal, aclaramiento de creatinina menor de 50 $\mathrm{mL} / \mathrm{min}$, hipersensibilidad conocida al fármaco, embarazo y lactancia $^{21}$

Un estudio no controlado con 53 pacientes con COVID-19 mostró mejoría clínica en dos tercios de los casos tratados con remdesivir; pero la ausencia de controles impide estimar la eficacia real ${ }^{23}$.

\section{Interferón alfa y beta}

Los interferones activan las enzimas citoplasmáticas que afectan la traducción del ARN mensajero viral y la síntesis proteica. El uso propuesto en COVID-19 se basa en trabajos in vitro y en observaciones clínicas en SARS, MERS y otras variedades de coronavirus, donde se ha documentado que la enfermedad está mediada tanto por la replicación viral como por la respuesta inflamatoria del anfitrión. Se han probado los dos tipos de interferón que mostraron actividad in vitro ${ }^{22,24-26}$. El estudio MIRACLE de Arabia Saudita evalúa la combinación de interferón beta con LPV/r en MERS-CoV ${ }^{26}$, y el ensayo de la OMS, Solidarity, también estudia esta combinación ${ }^{4}$.

\section{Los desafíos del uso off-label de los fármacos}

La indicación de un medicamento llamada "fuera de prospecto" u "off-label" se refiere a su utilización para una indicación distinta a aquellas para las que éste se encuentra oficialmente registrado y autorizado, en la medida en que no existan tratamientos alternativos, y que la evolución de la afección padecida signifique una amenaza para la salud $y / 0$ integridad $y / o$ vida de la persona que la padece 27,28

Existe mucha presión para usar fármacos candidatos como un "último recurso", bajo el supuesto de que los beneficios superarán a los daños. Cuando el uso es individual, no hay forma de saber si los pacientes se beneficiaron o se vieron perjudicados. Sin embargo, en el contexto de incertidumbre, su uso se ve impulsado pues se tiende a creer que, si el paciente fallece, es a causa de la infección, pero si se cura es debido al fármaco ${ }^{1}$. Así, sin un grupo control, no es posible determinar ninguna de las dos cosas.

Esto no solo implica un riesgo para los pacientes, sino que también estimula el uso indiscriminado, el desabastecimiento para su uso en las indicaciones aprobadas ${ }^{29}$ y, lo que resulta más preocupante, compromete la participación en estudios controlados que contribuyan a profundizar el conocimiento de la eficacia de las intervenciones propuestas.

En el pasado se ha observado como muchos fármacos, que in vitro parecían candidatos adecuados, fracasaron luego cuando se realizaron ensayos clínicos. Por otro lado, si bien utilizar medicamentos antiguos en forma off-label tiene la ventaja de que se conoce su perfil de seguridad, aún faltan datos que demuestren su eficacia. La mayor parte de los estudios de casos publicados hasta el momento, utilizando unos u otros medicamentos, carecen de un grupo de comparación pertinente ${ }^{1,2,30}$. Afortunadamente, y como se mencionó a propósito de cada fármaco evaluado, están en marcha protocolos diseñados con controles adecuados, que en el corto plazo permitirán caracterizar mejor su balance beneficio/riesgo, y de hecho, aunque con datos preliminares, se empiezan a publicar sus resultados ${ }^{10}$.

Aunque es muy difícil realizar ensayos controlados aleatorizados en el contexto de un brote, cualquier uso experimental de fármacos debería realizarse en un marco de investigación, con un protocolo definido, y una rigurosa recopilación e interpretación de los datos. Es de destacar el ensayo de Cao y $\mathrm{col}^{19}$, que aunque presenta resultados aparentemente negativos, muestra cómo es posible implementar diseños que permitan obtener resultados en forma rápida y permite a otros aprender de la experiencia, ya sea con valores positivos o negativos pero de buena calidad.

Como se mencionó dentro de las recomendaciones del MSN, las estrategias farmacológicas propuestas tienen baja calidad de evidencia, y por la naturaleza de su indicación fuera de prospecto, se requerirá el consentimiento informado ${ }^{27}$. En función de estas recomendaciones, y para garantizar el acceso a los tratamientos en el territorio nacional, se ha dispuesto desde el Sistema Nacional de Vigilancia de la Salud (SNVS 2.0), un sistema de registro de pacientes, para garantizar el registro de los resultados observados (Registro Arg-COVID) ${ }^{31}$. También se expresa el deseo de que "... Siempre que sea posible, el tratamiento debería estar enmarcado en un estudio clínico controlado..."2

El mega ensayo Solidarity ${ }^{4}$ comparará cuatro opciones de tratamiento (remdesivir; LPV/r; LPV/r con interferón beta-1a; y cloroquina o HCQ) con el estándar de atención, para evaluar su efectividad relativa contra COVID-19 considerando la necesidad de gran velocidad y escala en el ensayo (al 27 de marzo, más de 70 países habían confirmado su participación). Así se prevé que este estudio reducirá el tiempo necesario para evaluar los desenlaces de interes el $80 \%$ comparado con los ensayos clínicos controlados tradicionales ${ }^{1,3}$. Esto superará el riesgo de que múltiples ensayos pequeños no generen la evidencia sólida necesaria para determinar la efectividad relativa de los posibles tratamientos.

La International Society of Drug Bulletins nos recuerda que "A pesar de las buenas intenciones y la esperanza en conseguir un acceso rápido a medicamentos y vacunas contra el dañino virus COVID-19, debemos garantizar que los principios rectores de la medicina in dubio abstine (en caso de duda, abstenerse de tratar) y primum non nocere (lo primero, no hacer daño) no sean olvidados" (traducción de los autores) ${ }^{30}$

Es importante mantener la confianza de la poblacion, asegurando que todas las decisiones de salud pública se toman con las mejores pruebas disponibles, privilegiando el cuidado de la población en cualquier circunstancia y, a su vez, generando datos que permitan ratificar o rectificar oportunamente dichas decisiones.

\section{Cómo actuar sin dejar de aprender}

El planteo básico de la Medicina Basada en la Evidencia pedía integrar las mejores pruebas disponibles con la competencia clínica individual, la consideración del contexto, los valores y las preferencias del paciente ${ }^{32}$. Pero ¿cómo proceder cuando la situación es grave y la evidencia es de baja o muy baja calidad?

El juicio clínico debe partir de la consideración de la relación beneficio/riesgo en el paciente individual. Como los eventuales beneficios tienden a ser proporcionales a la gravedad del cuadro, mientras que los riesgos que derivan del tratamiento son más constantes, la relación resulta menos favorable en los casos más leves que en los más severos. Del mismo modo, si la comorbilidad del paciente aumenta sustancialmente el riesgo de efectos adversos graves, el beneficio incierto ya no justifica su uso.

El contexto de la pandemia en curso nos enfrenta a una avalancha de casos de una enfermedad con mortalidad significativa para la que no tenemos tratamiento específico. Es imprescindible articular las conductas individuales en proyectos que produzcan las respuestas que se necesitan.

Hoy más que nunca precisamos sistematizar las acciones y registrar sus resultados, para poder aprender de la experiencia. Para ello es necesario, dentro de lo posible, sumarse a los estudios en curso a nivel nacional e internacional.

El paciente tiene derecho a saber que el tratamiento específico que se le ofrece tiene carácter experimental, y a una presentación 
realista de los beneficios y los riesgos esperados. También tiene derecho a que su uso sea cuidadosamente supervisado y registrado, de modo que pueda contribuir a generar el conocimiento que se necesita con urgencia. Sólo así su consentimiento será realmente informado.

Frente a la conmoción global que genera la pandemia de COVID-19, se plantea la doble necesidad de actuar y de aprender en forma simultánea. Como señala Angus, en condiciones habituales esas dos tareas las llevan adelante equipos diferentes de profesionales, con métodos y criterios propios. La circunstancias obligan a integrar su trabajo y adaptar mutuamente sus estrategias $^{22,33}$.

Fuente de financiamiento/Conflicto de interés de los autores: Los autores no tuvieron financiamiento alguno para la preparación de este manuscrito. Durante la realización de este trabajo no hubo conflicto de intereses por parte de ninguno de los autores.

\section{Referencias}

1. Kalil AC. Treating COVID-19-Off-Label Drug Use, Compassionate Use, and Randomized Clinical Trials During Pandemics. JAMA. 2020;Available from: 10.1001/jama.2020.4742.

2. Canada, British Columbia, Ministry of Health Centre For Disease Control. Unproven Therapies for COVID-19; 2020. Available from: https://bit.ly/ 2X16PX4 [Last access: 2020-04-16].

3. Argentina Ministerio De Salud. Recomendaciones condicionales para el abordaje terapéutico de COVID-19; 2020. Available from: https://www. argentina.gob.ar/salud/coronavirus-COVID-19/abordaje-terapeutico [Last access: 2020-04-16].

4. World Health Organization. Solidarity clinical trial for COVID-19 treatments; 2020. Available from: https://www.who.int/emergencies/diseases/novelcoronavirus-2019/global-research-on-novel-coronavirus-2019-ncov/solidarity-clinical-trial-for-covid-19-treatments [Last access: 2020-04-16].

5. Argentina Ministerio de Salud Red Argentina Pública de Evaluación de Tecnologías Sanitarias (RedARETS). Informe Rápido de Evaluación de Tecnología Sanitaria (IRETS): Tratamiento farmacológico específico con cloroquina en caso de infección por COVID-19; 2020. Available from: http://www.redarets.com.ar/index.php/component/phocadownload/category/4-archivos?download=14:irets-cloroquina-03-2020 [Last access: 202004-16].

6. Cortegiani A, Ingoglia G, Ippolito M, et al. A systematic review on the efficacy and safety of chloroquine for the treatment of COVID-19. J Crit Care. 2020;S0883-9441(10):30390-7. Available from: 10.1016/j.jcrc.2020.03.005.

7. Chen J, Liu D, Liu L, et al. A pilot study of hydroxychloroquine in treatment of patients with common coronavirus disease-19 (COVID-19). J Zhejiang Univ (Med Sci). 2020;49(1):0-0. Available from: 10.3785/j.issn.1008-9292.2020.03.03;http://www.zjujournals.com/med/EN/10.3785/j.issn. 1008-9292.2020.03.03.

8. Gautret P, Lagier JC, Parola P, et al. Hydroxychloroquine and azithromycin as a treatment of COVID-19: results of an open-label non-randomized clinical trial. Int J Antimicrob Agents. 2020;Available from: 10.1016/j.ijantimicag.2020.105949.

9. Prescrire. Covid-19 et essais de médicaments : que faire des premiers résultats d'évaluation?; 2020. Available from: https://bit.ly/39ons1w [Last access: 2020-04-16]

10. Voss A. Statement on IJAA paper. Official Statement from International Society of Antimicrobial Chemotherapy (ISAC). Hydroxychloroquine and azithromycin as a treatment of COVID-19: results of an open-label non-randomized clinical trial (Gautret P et al. PMID 32205204); 2020. Available from: https://www.isac.world/news-and-publications/official-isac-statement [Last access: 2020-04-16].

11. Gautret P, Lagier JC, Parola PE, et al. Clinical and microbiological effect of a combination of hydroxychloroquine and azithromycin in 80 COVID-19 patients with at least a six-day follow up: an observational study. Travel Med Infect Dis. 2020;Available from: 10.1016/j.tmaid.2020.101663.

12. Chen Z, Hu J, Zhang Z, et al. Efficacy of hydroxychloroquine in patients with COVID-19: results of a randomized clinical trial. MedRvix. 2020;Available from: 10.1101/2020.03.22.20040758; https://www.medrxiv.org/content/10.1101/2020.03.22.20040758v3.

13. Mahevas M, Tran VT, Roumier M, et al.. No evidence of clinical efficacy of hydroxychloroquine in patients hospitalized for CoVID-19 infection with oxygen requirement: results of a study using routinely collected data to emulate a target trial; 2020. Available from: https://www.medrxiv.org/content/ 10.1101/2020.04.10.20060699v1 [Last access: 2020-04-17].

14. Tang W, Cao Z, Han M. Hydroxychloroquine in patients with COVID-19: an open-label, randomized; 2020. Available from: https://www.medrxiv.org/ content/10.1101/2020.04.10.20060558v1 [Last access: 2020-04-17].

15. Silva-Borba MG, de Almeida-Val F, Sousa-Sampaio V, et al.. Chloroquine diphosphate in two different dosages as adjunctive therapy of hospitalized patients with severe respiratory syndrome in the context of coronavirus (SARS-CoV-2) infection: Preliminary safety results of a randomized, double-blinded, phase Ilb clinical trial (CloroCovid-19 Study); 2020. Available from: https://www.medrxiv.org/content/10.1101/2020. 04.07.20056424v2.article-metrics? cf chl jschl tk =d4a9b4e7b02567bb9207f749a288266120fead9e-1587139034-0-AY4RZm3pt1wckYrT0SYfoDKbFI7UkCwyV33KreCVG2QCm76tgKVFFHjFOi2lrulfWM VLyHJqSt8toCplJbuNgRjHX9kRV7SXDP9ghuWWqpPEK-7unjmLCqh4Jw11 ZnG3fssbkdaJH0I3d1dcno9K5mtlOOyx-FiE17v_rscgWFUZUu-SIUXmL6uJL3UV-tyltQ8ddfrRoVQZt9YABLs-9QXHY9JNV8T4GtC4DtEeOdQW -P4VXXtqWpeWD-nYI6vOVqNLkE_XV1Ukuspru0epKWqT3j3NUADhVbaGGD-xSpkweQrWrEq_Ep_npf0wA8YIE8uAgX00RzmY1aug2tHeAnJSoDgUCFL5xIrkDHF [Last access: 2020-04-17]

16. Mena MB, Durán C, Marchand B. Cloroquina e hidroxicloroquina no han demostrado ser eficaces en el tratamiento de pacientes con Covid-19; 2020. Available from: http://www.proyectodime.info/documents/127/Excellencis-Cloroquina e Hidroxicloroquina no han demostrado ser_eficaces.pdf [Last access: 2020-04-16].

17. Sociedad Argentina de Terapia Intensiva Comité de Patología Crítica Cardiovascular. Informe Cloroquina o Hidroxicloroquina; 2020. Available from: https://www.sati.org.ar/images/2020-03-26-COVID-19_Cloroquina_Hidroxicloroquina-COMITE_Patologia_Critica_CV.pdf [Last access: 202004-16].

18. Red Argentina Pública de Evaluación de Tecnologías Sanitarias (RedARETS). Informe Rápido de Evaluación de Tecnología Sanitaria (IRETS): Tratamiento farmacológico específico con lopinavir/ritonavir de pacientes con infección por COVID-19; 2020. Available from: http://www.redarets.com.ar/index.php/component/phocadownload/category/4-archivos?download=16:informe-rapido-de-evaluacion-de-tecnologiasanitaria-irets-tratamiento-farmacologico-especifico-de-pacientes-con-infeccion-por-covid-19 [Last access: 2020-04-16].

19. Cao B, Wang Y, Wen D, et al. A trial of lopinavir-ritonavir in adults hospitalized with severe Covid-19. N Engl J Med. 2020;Available from: 10.1056/ NEJMoa2001282.

20. Young BE, Ong S, Kalimuddin S, et al. Epidemiologic Features and Clinical Course of Patients Infected With SARS-CoV-2 in Singapore. JAMA. 2020;2020:16-22. Available from: 10.1001/jama.2020.3204.

21. España Ministerio de Sanidad Agencia Española de Medicamentos y Productos Sanitarios. Tratamientos disponibles para el manejo de la infección respiratoria por SARS-CoV-2; 2020. Available from: https://bit.ly/3evnB7e [Last access: 2020-04-17].

22. Sheahan TP, Sims AC, Leist SR, et al. Comparative therapeutic efficacy of remdesivir and combination lopinavir, ritonavir, and interferon beta against MERS-CoV. Nat Commun. 2020;11(1):222. Available from: 10.1038/s41467-019-13940-6.

23. Grein J, Ohmagari N, Shin D, et al. Compassionate Use of Remdesivir for Patients With Severe Covid-19. N Engl J Med. 2020;Available from: 10.1056/NEJMoa2007016.

24. Falzarano D, Wit E, Martellaro $\mathrm{C}$, et al. Inhibition of novel $\beta$ coronavirus replication by a combination of interferon- $\alpha 2 b$ and ribavirin. Sci Rep. 2013;3(1):1686-1686. Available from: 10.1038/srep01686. 
25. Chan JF, Yao Y, Yeung ML, et al. Treatment With Lopinavir/Ritonavir or Interferon- $\beta 1 \mathrm{~b}$ Improves Outcome of MERS-CoV Infection in a Nonhuman Primate Model of Common Marmoset. J Infect Dis. 2015;212(12):1904-1917. Available from: 10.1093/infdis/jiv392.

26. Arabi YM, Asiri AY, Assiri AM, et al. Treatment of Middle East respiratory syndrome with a combination of lopinavir/ritonavir and interferon- $\beta 1 \mathrm{~b}$ (MIRACLE trial): statistical analysis plan for a recursive two-stage group sequential randomized controlled trial. Trials. 2020;21(1):8. Available from: 10.1186/s13063-019-3846-x.

27. Argentina Ministerio de salud de la Nación. Modelo de consentimiento Informado para el tratamiento farmacológico de pacientes con COVID-19 (coronavirus); 2020. Available from: https://www.argentina.gob.ar/sites/default/files/covid-19 modelo-consentimiento-informado.pdf [Last access: 2020-04-16].

28. World Health Organization. Off-label use of medicines for COVID-19; 2020. Available from: https://www.who.int/news-room/commentaries/detail/offlabel-use-of-medicines-for-covid-19 [Last access: 2020-04-16].

29. Red Argentina de Centros de Información de Medicamentos (RACIM). Disponibilidad de medicamentos: llamado a la solidaridad; 2020. Available from: https://www.fundacionfemeba.org.ar/blog/farmacologia-7/post/racim-disponibilidad-de-medicamentos-con-hidroxicloroquina-llamado-ala-solidaridad-47678 [Last access: 2020-04-16]

30. International Society of Drug Bulletins. COVID-19 and the quest for drugs and vaccines: Statement from the International Society of Drug Bulletins 2020. Available from: https://www.isdbweb.org/publication/press-release/statement-covid-19 [Last access: 2020-04-16]

31. Argentina Ministerio De Salud De La Nación. Acceso a medicación antiviral para COVID-19; 2020. Available from: https://www.argentina.gob.ar/ recomendaciones-para-equipos-de-salud/acceso-medicacion-antiviral-para-covid-19 [Last access: 2020-04-16]

32. Sackett DL, Rosenberg WMC, Gray JAM, et al. Evidence based medicine: what it is and what it isn't. BMJ. 1996;312(7023):71-72. Available from: 10.1136/bmj.312.7023.71.

33. Angus DC. Optimizing the Trade-off Between Learning and Doing in a Pandemic. JAMA. 2020;Available from: 10.1001/jama.2020.4984. 